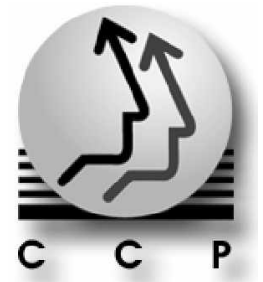

Población y Salud en Mesoamérica

Revista electrónica publicada por el

Centro Centroamericano de Población

Universidad de Costa Rica, 2060 San José, Costa Rica

http://ccp.ucr.ac.cr

Población y Salud en Mesoamérica

Revista electrónica semestral, ISSN-1659-0201

Volumen 5, número 2, artículo 3

Enero - junio, 2008

Publicado 1 de enero, 2008

http://ccp.ucr.ac.cr/revista/

\title{
Fortalezas y Debilidades del Proyecto de Atención a Poblaciones Móviles para la disminución del VIH- SIDA en El Salvador, una intervención de campo
}

\author{
Helen Larissa Centeno Monge
}

(C) 2008 Centro Centroamericano de Población 


\title{
Fortalezas y Debilidades del Proyecto de Atención a Poblaciones Móviles para la disminución del VIH-SIDA en El Salvador, una intervención de campo
}

\author{
Strengths and Weaknesses of the Project of Attention to Mobile Populations for the \\ diminution of the VIH-SIDA in El Salvador, a field intervention project
}

\author{
Helen Larissa Centeno Monge ${ }^{I}$
}

\section{RESUMEN}

Este artículo describe algunas de las fortalezas y debilidades encontradas en el Proyecto de Atención a Poblaciones Móviles y sectores que las atienden directamente: personal de salud, migración, aduanas, cuerpos uniformados recién instalados en las fronteras a nivel nacional plasmado en el Plan Estratégico Nacional para la Prevención, Atención y Control del VIH-SIDA e ITS / El Salvador 2005 -2010 El propósito de la intervención fue desarrollar la capacidad, mejorar conocimientos y habilidades para realizar actividades de educación e información continuas para desarrollar una percepción de riesgo y un cambio hacia conductas sexuales seguras o de menor riesgo ante las ITS/VIH/SIDA y así incidir en la disminución de su transmisión. La metodología incluyó tres momentos claves: la construcción de una línea de base, la implementación de talleres educativos y de información y una evaluación final. Logró captar un total de 380 individuos a nivel nacional. De los resultados mas significativos se pueden mencionar los alcanzados por los participantes que no pertenecían al sector salud, los cuales evidencian un aumento de conocimientos básicos transmisión y prevención del VIH- y adquisición de habilidades de un $40 \%$ hasta un $60 \%$ más de lo encontrado en la línea basal, situación que no resultó ser la misma en los profesionales de salud, en quienes se observo una tendencia similar en la línea basal y en la evaluación final. Se concluye que los esfuerzos realizados desde que dan inicio las intervenciones en poblaciones móviles en el año 2001 y que tienen como fin frenar el avance del SIDA en El Salvador no han sido suficientes hasta el momento por carecer de participación activa de todos los sectores que atienden a dichas poblaciones vulnerables y expuestas a riesgo.

Palabras clave: Poblaciones móviles, grupos vulnerables, incidencia de VIH-SIDA, emigrantes en riesgo.

\begin{abstract}
This article describes some of the strengths and weaknesses found in the Project of Attention to Movable Populations and sectors that take care of them directly: personnel of health, migration, customs, bodies uniformed just installed in the borders at shaped national level in the National Strategic Plan for the Prevention, Attention and Control of HIVAIDS and ITS/El Salvador 2005 - the 2010. The intention of the intervention was to develop the capacity, to improve knowledge and abilities to make activities of continuous education and information to develop to a perception of risk and a change towards safe sexual conducts or of smaller risk before its/VIH/SIDA and thus of affecting the diminution of its transmission. The methodology included three key moments: the construction of a base line, the implementation of educative factories and information and a final evaluation. It managed to catch a total of 380 individuals at national level. Of the significant results that can be mentioned are the reached ones about the participants who did not belong to the sector health, which demonstrate an increase of basic knowledge transmission and prevention of the HIV- and acquisition of abilities of a $40 \%$ until a $60 \%$ more of the found thing in the basal line, situation that did not turn out to be the same one in the health professionals, in those who we observed a similar tendency in the basal line and the final evaluation. One concludes that the made efforts since they give to beginning the interventions in movable populations in 2001 and that they must like aim stop the advance of the AIDS in El Salvador have been not sufficient until the moment for lacking active participation of all the sectors that take care of these vulnerable and exposed risk populations.
\end{abstract}

Keywords: Movable population, vulnerable groups, incidence of HIV-AIDS, emigrants in risk.

Recibido: 11 set. 2007

Aprobado: oct. 2007

\footnotetext{
${ }^{1}$ Coordinadora Diplomados y Postgrados de VIH-SIDA del programa PNUD - Unidad de Maestría en Salud Sexual y Reproductiva, Facultad de Medicina, Universidad de El Salvador, EL SALVADOR. lariscen@yahoo.com
} 


\section{INTRODUCCION}

El Síndrome de Inmuno Deficiencia Adquirida (SIDA) es una de las enfermedades mas devastadoras de la historia reciente, y que indudablemente ha causado un gran impacto en la vida moderna, evidenciada principalmente en los países en vías de desarrollo, especialmente en los indicadores que miden la calidad de vida de una población.

En El Salvador el primer caso de SIDA se diagnosticó en 1984 y desde ese momento los casos acumulados registrados se han incrementado sostenidamente hasta la actualidad alcanzando a febrero de 2007 la cifra de 18,284 ${ }^{2}$. Las estimaciones realizadas por el Programa Conjunto de las Naciones Unidas dedicado al Virus de Inmunodeficiencia Humana (VIH) conocido por sus siglas ONUSIDA indicaron que para el 2010 serían 81,904. Estas cifras indican que cada día se estarían infectando de 11 a 21 personas y morirían a consecuencia de la enfermedad de 4 a 8 salvadoreños ${ }^{3}$.

Tomando en cuenta la relación entre población que se desplaza y el VIH y el SIDA, hay que hacer mención que la migración, la movilidad y el VIH-SIDA son importante fenómenos mundiales del comienzo del nuevo milenio. De hecho se podría decir que ha sido uno de los factores explicativos más importantes en los modelos que se han aplicado para estudiar epidemiológicamente la difusión del SIDA en América Latina y especialmente en Centroamérica, pues la vulnerabilidad de las personas móviles ante el virus actualmente se considera como uno de los determinantes más importantes del riesgo de transmisión. Entendiéndose como Población Móvil aquellas personas que se desplazan de un lugar a otro temporalmente, estacionalmente o definitivamente por un conjunto de razones voluntarias o involuntarias ${ }^{4}$.

En El Salvador el 25\% de la población son migrantes externos. En el 2002 hubo 3,500,000 movimientos migratorios, tendencia que ha venido en franco aumento desde 1978, reduciéndose en 1994 y 1995; luego recuperando la tendencia ascendente hasta la fecha. Se estima que del total de los movimientos migratorios, casi 2,000,000 son nacionales, $1,100,00$ son centroamericanos a predominio de Nicaragüenses y Hondureños y 600,000 son extranjeros del resto del mundo.

La experiencia de 6 años trabajando con poblaciones móviles, el estudio a profundidad de los contextos de riesgo y el diagnóstico situacional de la realidad del país en materia de VIH-SIDA ${ }^{5}$ ha puesto sobre la mesa una seria problemática y determina dos situaciones preocupantes e importantes: uno que El Salvador es un corredor de personas de un país a otro y dos que no existe en él recurso humano en las fronteras que este capacitado e informado, que promueva y participe de acciones continuas de información sobre la prevención del VIH-SIDA

El diagnostico situacional indicó que los esfuerzos realizados para frenar el avance de lo que se ha llamado la epidemia del SIDA han sido insuficientes hasta el momento debido a que no se ha contado con la participación y el involucramiento de las poblaciones expuestas a riesgo.

\footnotetext{
2 Programa Nacional de ITS/VIH-SIDA, MSPAS

${ }^{3}$ Informe Anual ONUSIDA 2006

${ }^{4}$ Guía de Atención Integral a Poblaciones Móviles, El Salvador

${ }^{5}$ Programa Nacional de ITS/VIH-SIDA, MISPAS
} 
El Ministerio de Salud Pública y Asistencia Social y otras ONG's conocedoras de la importancia de la relación entre las poblaciones móviles no atendidas y la expansión del VIH-SIDA, inicia a partir de 2001 esfuerzos focalizados para el abordaje y atención de estas poblaciones en dos estaciones de paso, y a partir del 2004 lanza la iniciativa de Prevención del VIH-SIDA en Poblaciones Móviles a todas las fronteras terrestres, aéreas y marítimas en El Salvador. Y no es sino hasta el 2006 que lanza una iniciativa para fortalecer los conocimientos sobre esta epidemia en sectores que trabajan atendiendo día con día a las poblaciones móviles: cuerpos uniformados, personal de aduanas y migración recién incorporados de ambos lados de las fronteras terrestres a través de intervenciones de prevención de alto impacto por lo que se realizaron talleres de Información, Educación y Comunicación efectiva (IEC) dirigidos a dicho personal, entre otras. De tales acciones se esperaba mejorar los conocimientos de las personas, y generar actitudes para el cambio hacia prácticas sexuales protegidas o de menor riesgo en esta población, que pudiesen influir en la disminución de la transmisión del VIH-SIDA.

Cabe mencionar que ya existen acuerdos entre El Salvador, Guatemala y Honduras para trabajo conjunto, en áreas fronterizas, que dan marco a estas intervenciones y que específicamente suscribe acuerdo de trabajo conjunto en la prevención del VIH-SIDA ${ }^{6}$.

Partiendo de la anterior visión, el presente articulo constituye un enfoque del Proceso de Capacitación desarrollado, en espera de que esta información se convierta en una herramienta de evaluación, seguimiento del proceso y en una posibilidad de respuesta efectiva a las necesidades educativas que se demandan para la Atención Integral de las Poblaciones Móviles.

\section{DATOS Y METODOS}

Se utilizan datos obtenidos durante la Intervención de campo desarrollada durante los meses de Mayo y Julio de 2006, en jornadas de 16 horas distribuidas en dos días, en los trece puestos fronterizos de El Salvador. Se incluyen fronteras terrestres que colindan con Honduras y Guatemala, Puertos Marítimos, Estaciones de Paso y Puerto aéreo (ver figura 1). El Proceso tenía un alcance de 400 individuos a nivel nacional pertenecientes a los sectores de salud, migración, aduanas y cuerpos uniformados y cuerpo naval recién incorporados en dichos puestos fronterizos, así como líderes comunitarios de las cercanías, de los cuales hubo una deserción del 5\% (20 personas). Del total final de población captada el $76 \%$ eran del sexo masculino (288) y $24 \%$ del sexo femenino (92).

Para valorar los logros alcanzados en toda la población, la metodología incluyo tres pasos:

- PRE TEST: fundamental para la creación de un diagnostico situacional- línea de base- lo cual fue un instrumento valioso porque facilito la identificación de vacíos conceptuales básicos relacionados con el VIH-SIDA, mitos y tabúes preexistentes, y necesidades de aprendizaje, entre otros.

\footnotetext{
${ }^{6}$ Articulo 24 del Plan Estratégico Nacional para la Prevención, Atención y Control del VIH-SIDA e ITS /2005 2010,
} 
- CAPACITACIÓN-TALLER: la Metodología aplicada consistió en el desarrollo de la capacitación a través de talleres educativos y de información los cuales se convirtieron en el espacio en el que no solo se adquirieron o reafirmaron conocimientos y habilidades, sino también, se posibilitó un proceso planificado de Enseñanza-Aprendizaje con temáticas especificas como epidemiología mundial, regional y local, conceptos básicos, transmisión, prevención y uso correcto y consistente del condón a su vez que permitió el desarrollo de actitudes y valores requeridos para cumplir con los objetivos de la intervención y con el cometido de una misión específica: la contribución en la disminución de la incidencia del VIH-SIDA en poblaciones móviles, vulnerables y con alto riesgo.

- POST TEST: realizado al finalizar el proceso de capacitaciones, para medir objetivos expectativas alcanzadas. Para el análisis de los datos se utilizan los obtenidos durante el pretest y postest.

El proceso desarrollado en estos tres pasos y el aprendizaje concebido de esta forma se encuentra relacionado estrechamente con los problemas concretos de la cotidianidad en el tema de VIHSIDA, vinculado a las acciones de Atención a las Poblaciones Móviles. Es importante la participación del personal de salud, personal uniformado, personal de migración y aduanas bifroterizos y líderes comunitarios, quienes forman parte del elemento dinamizador en el proceso. La interrelación del grupo con la experiencia y otras fuentes documentales del conocimiento hacen énfasis en el proceso, alejado de las charlas y conferencias puntuales de especialistas.

Esto implicó la creación de un clima amplio de auto expresión, de análisis y reflexión que condujo a la búsqueda personal crítica y autocrítica y a una adecuada sensibilización ante la epidemia del VIH, y a fortalecer y sensibilizar sobre el riesgo al que todos estamos expuestos. Sin olvidar por ello que este tipo de metodología también fortalece los conocimientos en derechos humanos haciendo hincapié en la discriminación social y el estigma a los que se han visto sometidos los portadores del virus, aun hoy en día.

\section{RESULTADOS}

\subsection{Confiabilidad de los resultados}

Para efectos de presentar los resultados, y teniendo en cuenta que no todas las personas que completaron el pre-test, completaron un post-test, pues no concluyeron el proceso (deserción de un 5\%) se tomo la decisión de realizar un muestreo al azar de los participantes al finalizar la intervención, seleccionando $50 \%$ de las personas del área de salud y un 50\% de las personas de migración, aduanas, cuerpos uniformados y líderes comunitarios- denominados "el resto".

Al momento de la selección se tomaron dos criterios de inclusión:

- Participante que cumplió con las 16 horas de capacitación

- Eminentemente perteneciente a la población meta 
Para medir conocimientos previos y los alcanzados al finalizar la intervención se consideraron aquellas preguntas que incluían conceptos básicos, mecanismos de transmisión y formas de prevención del VIH-SIDA, lo cual permitió hacer un análisis comparativo (ver cuadro 1).

\subsection{Prevalencia}

De acuerdo al análisis comparativo realizado en la población finalmente captada por la intervención de campo, con respecto a buenos conocimientos básicos sobre VIH-SIDA, la prevalencia es mayor en las personas pertenecientes al área de salud, y la disminución de Estigma y discriminación hacia personas viviendo con el virus y poca co-existencia de mitos y tabúes relacionados también se refleja en el mismo sector. Un resultado que se torna preocupante es la poca información y la elevada prevalencia sobre algunos mitos y tabúes en la población denominada "el resto" que en algunos casos alcanzan hasta un 50\% de respuestas erradas.

\section{DISCUSION}

Se tomo en cuenta en el analisis de los datos, que algunas de las diferencias bien marcadas entre el personal de salud y el "resto" se debe al constante proceso de capacitaciones al que se ve sometido dicho personal como parte del del Plan estrategico en lucha contra el VIH-SIDA a nivel nacional, coordinado por el Promagrama Nacional de VIH-SIDA y algunas ONG's que trabajan con la tematica.

En las tres grandes areas exploradas con el pre y post test: conocimientos de conceptos básicos, estigma y discriminación y mitos y tabues sobre el VIH-SIDA cabe señalar que el personal de salud obtuvo los mejores resultados, pues las diferencias en ambos momentos fueron minimas o nulas con respecto a las respuestas correctas. A diferencia del "resto" quien mostró un evidente aumento de respuestas correctas al finalizar el proceso.

En el primer bloque de preguntas encaminadas a evaluar los conocimientos básicos sobre el VIHSIDA, para el personal de salud no significo mayor dificultad, ya que en promedio el $100 \%$ reflejo respuestas correctas, a diferencia del "resto" en quienes se observo variabilidad de respuestas, que fueron desde un $90 \%$ de respuestas incorrectas en la linea de base, que fueron superadas en un 40 a $60 \%$ al final del proceso. (gráficos 1 Y 2)

En el conjunto de preguntas encaminadas a medir niveles de estigma y discriminación persistentes hacia las personas viviendo con VIH se pudo observar que el personal de salud, ante la pregunta de disponibilidad para cuidar en su casa a un familiar infectado con VIH, un $62 \%$ respondio que "si" en el pretest disponibilidad que aumento a un $92 \%$ en la evaluación final. En "el resto" en cambio, para las mismas preguntas hubo un aumento en la disponibilidad y disminución de la discriminación que fue desde un 16-32\% en el pretest hasta un 63-84\% en el post test.

En el resto de preguntas relacionadas a la disponibilidad de ser multiplicadores de los conocimientos adquiridos tanto en sus comunidades como en los lugares de trabajo, los resultados fueron bastante similares en ambos grupos y evaluaciones, reflejando un $100 \%$ de 
disponibilidad despues de terminado el proceso. Esto denota que con la información correcta es posible trabajar con la temática en diversos ámbitos y convivir en diferentes espacios con aquellas personas sero positivas. (gráficos $3 \mathrm{Y} 4$ )

En cuanto a la persistencia de mitos y tabues relacionados con el VIH-SIDA, se hace más evidente en el personal que trabaja en aduanas, migración, cuerpos uniformados y lideres de la comunidad -denomidos "el resto" en este articulo-, a diferencia de la tendencia presentada por el personal de salud, en quienes se ha logrado dismitificar a lo largo de estos 23 años de existencia de la epidemia en el país. Así podemos observar que en el bloque de preguntas referidos a "mitos y tabues" los porcentajes de respuestas correctas en el pre test son bien altos en el personal de salud a diferencia del "resto", pues en el pretest las personas que obtuvieron respuestas correctas en el promedio de las doce preguntas que incluia este apartado solo alcanzan un 40\% (76 personas) del 100\% de la población (190 personas). Situación que se modifica significativamente en un $50 \%$ al final del proceso, puesto que un promedio de 160 personas participantes lograron respuestas correctas que reflejan la dismitificación de la problemática del VIH-SIDA en El Salvador, y un mejor entendimientos de los tabues relacionados con la epidemia. (gráficos 5 Y 6 )

\section{CONCLUSIONES}

No obstante se ha avanzado mucho en la diseminación de información sobre la prevención del VIH-SIDA tanto a nivel de profesionales y técnicos de la salud, voluntarios y personas de la comunidad, es mucho lo que debe hacerse para que alcance el impacto esperado sobre el comportamiento de las personas vulnerables a la epidemia.

Este tipo de intervención de campo en poblaciones móviles y sectores que los atienden directamente y la implementación de la metodología participativa a través de múltiples capacitaciones-talleres de IEC en los trece puestos fronterizos de El Salvador, resulta efectivo en relación al reforzamiento, fortalecimiento y adquisición de conceptos básicos del VIH-SIDA, disminución de estigma y discriminación, y resolución de mitos y tabúes pre-existentes en gran parte de esta población salvadoreña, y podrían ser procesos continuos y sostenibles para aunar a los esfuerzos ya realizados.

Los esfuerzos realizados desde que dan inicio las intervenciones en poblaciones móviles en el año 2001 y que tienen como fin frenar el avance del SIDA en El Salvador, no han sido suficientes hasta el momento por haber carecido de la participación activa de todos los sectores que atienden a dichos sectores vulnerables y expuestos a riesgo, por lo que haber incursionado por primera vez en esta población debería servir de incentivo para gestionar procesos similares en el futuro.

Es importante fortalecer adecuadamente los conceptos relacionados a la prevención, específicamente la abstinencia, la fidelidad mutua y especialmente los mitos sobre el uso del preservativo, y realizar actividades que vayan encaminadas a disminuir estigma y discriminación hacia las personas viviendo con el VIH, aun presentes en gran parte de la sociedad salvadoreña.

El tema causa mucho interés y motivación en la población general. Es importante considerar el adecuado entrenamiento de profesionales reconocidos y líderes a nivel local que mantengan ese 
nivel de interés, aclaren dudas sobre el tema en los diferentes sectores de la comunidad, coordinen y gestionen acciones intersectoriales.

\section{RECOMENDACIONES}

Gestionar fondos con agencias Internacionales que permitan darle sostenibilidad a proyectos similares, para que no queden frustrados los esfuerzos logrados y para realizar monitoreo y evaluación semestralmente de los alcances de objetivos obtenidos durante los talleres.

Fortalecer el liderazgo del nivel local y su capacidad de gestión y convocatoria en la comunidad.

Fortalecer los aspectos conceptuales que tuvieron resultados insatisfactorios durante esta intervención de campo a nivel nacional.

Establecer un diagnóstico de necesidades educativas/capacitación, en el que se consideren las necesidades de acuerdo al perfil socio-cultural y educativo de personas a capacitar, tanto a nivel de la comunidad como del personal de salud.

Desarrollar habilidades en el personal de salud y personal de migración, aduanas, cuerpos uniformados y lideres comunales de los diferentes puestos fronterizos salvadoreños, para el adecuado desempeño del rol de promotores de conductas y actividades saludables en la población.

Desarrollar actividades de IEC con grupos específicos identificados o conocidos como de "mayor riesgo y vulnerabilidad" como lo constituyen las poblaciones móviles en la comunidad con metodología de cascada dirigidos y coordinados por el personal de salud del nivel local. 


\section{BIBLIOGRAFÍA}

ASAPROSAR (2006). Informe Final de Capacitación a Poblaciones Móviles. San Salvador: ASAPROSAR.

El Salvador. Ministerio de Salud Pública y Asistencia Social. Programa Nacional de ITS/VIHSIDA (2005). Guía de Atención para Poblaciones Móviles para el Control y Prevención de ITS/VIH/SIDA. San Salvador: El Ministerio.

El Salvador. Ministerio de Salud Pública y Asistencia Social. Programa Nacional de ITS/VIHSIDA (2006). La Lucha Contra el SIDA en El Salvador, Un Compromiso de Nación. San Salvador: El Ministerio.

El Salvador. Ministerio de Salud Pública y Asistencia Social. Programa Nacional de ITS/VIHSIDA (2005). Plan Estratégico Nacional para la Prevención, Atención y Control del VIHSIDA e ITS, 2005-2010. San Salvador: El Ministerio.

El Salvador. Ministerio de Salud Pública y Asistencia Social. Programa Nacional de ITS/VIHSIDA (2007). Situación Epidemiológica del VIH-SIDA en El Salvador, Años 1984-2007. San Salvador: El Ministerio.

Lopez, Ivette (2004). Plan Internacional. Guía para los Talleres de Sensibilización en VIHSIDA. Quito: Plan Oficina Regional Ecuador

Martínez, Jorge (2001). Poblaciones Móviles y VIH/SIDA en Centroamérica, México y Estados Unidos. Estación de paso: Corredor Interfronterizo Santa Ana, El Salvador-Jutiapa. San Salvador: IMUE - Universidad de El Salvador.

ONUSIDA. Programas Internacionales y Nacionales contra el SIDA (2006). Situación de la Epidemia de SIDA. San Salvador: ONUSIDA.

Organización Panamericana para la Salud (2006). Terminología Actualizada con el VIH: actualización de 2006. Washington: OPS

Tiley-Gyado, Abiola (1998). Plan Internacional Regional Office. Protocol for HIV/AIDS training for Plan Internacional Staff. Bolivia: Editorial Plan-Oficina Regional para las Américas. 
Figura 1. Mapa de EI Salvador: puntos fronterizos donde se realizan intervenciones en población móvil y migrantes

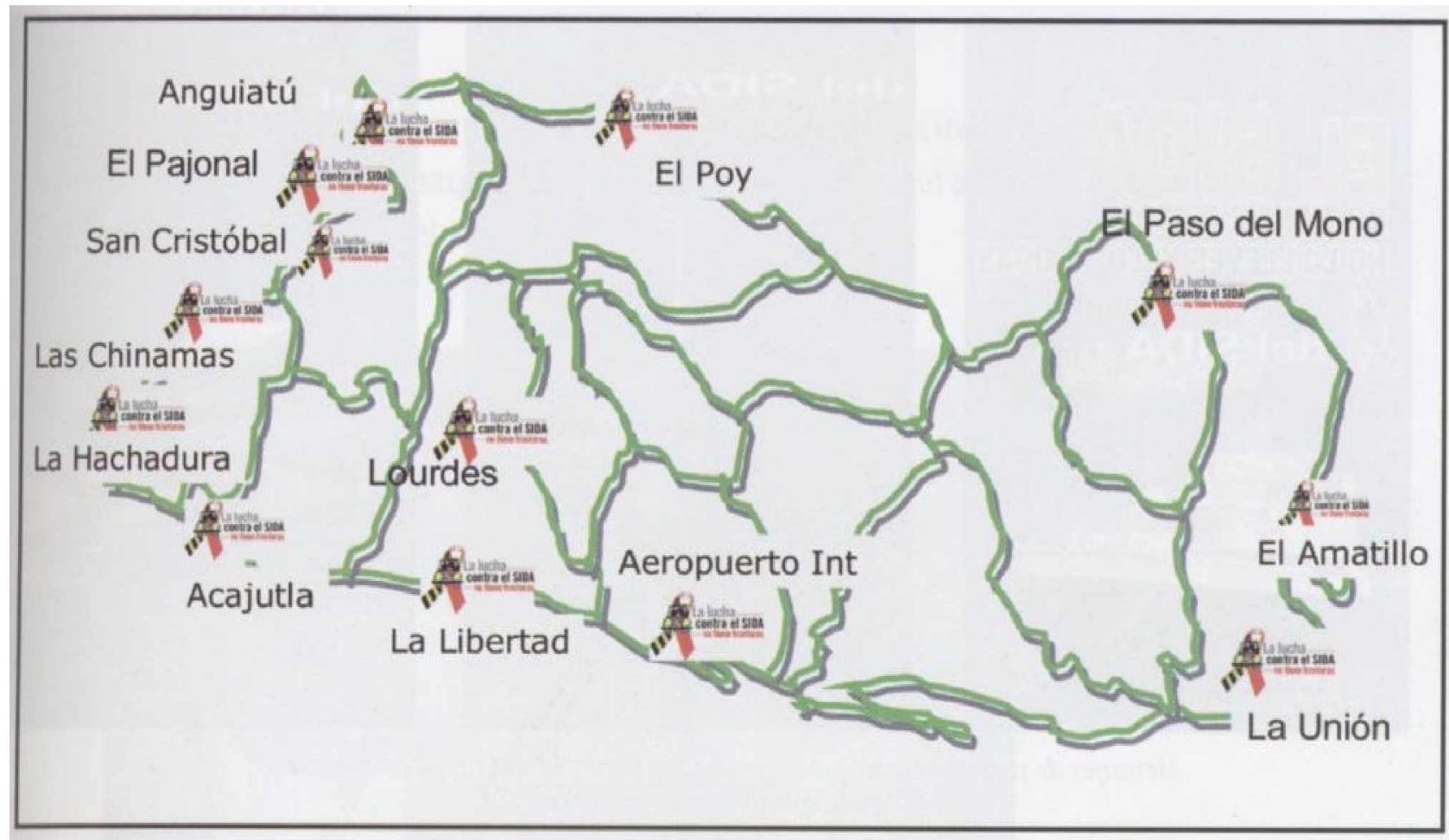

Mapa de El Salvador, señalando los puntos fronterizos donde se realizan intervenciones en población móvil y migrantes.

Fuente: La Lucha Contra el SIDA en El Salvador, Un compromiso de nación, pag.39 


\section{Cuadro 1. Preguntas incluidas para el análisis pre y post test}

\section{Conocimientos}

\begin{tabular}{|l|}
\hline VIH es lo mismo que SIDA \\
\hline Una persona que se ve sana puede trasmitir el VIH \\
\hline $\begin{array}{l}\text { Una persona infectada con el VIH, pero que aun no sufre de SIDA, puede trasmitir el } \\
\text { virus a otra persona }\end{array}$ \\
\hline $\begin{array}{l}\text { Puede trasmitirse el VIH/SIDA si el hombre eyacula afuera durante una relación sexo- } \\
\text { coital }\end{array}$ \\
\hline El condón disminuye el placer sexual \\
\hline El condón puede hacer que el hombre pierda la erección del pene \\
\hline El condón causa mal de orín \\
\hline
\end{tabular}

\section{Estigma y discriminación}

Si un pariente suyo se enfermera de SIDA, ¿estaría usted dispuesto/a a recibirlo y cuidarlo en su casa?

Si usted supiera que un(a) vendedor (a) de alimentos y verduras tiene SIDA, ¿le compraría?

Me sentiría cómodo (a) abordando temas de sexualidad y VIH/SIDA en mi comunidad

Me sentiría cómodo siendo multiplicador de los conocimientos que adquiera en este taller?

\section{Transmisión y prevención}

\begin{tabular}{|l|}
\hline El VIH se trasmite al dar la mano a una persona que lo posea \\
\hline $\begin{array}{l}\text { El VIH se trasmite al estar en contacto diario en el trabajo o en la comunidad con } \\
\text { personas que lo tienen }\end{array}$ \\
\hline $\begin{array}{l}\text { El VIH se trasmite al usar los mismos inodoros, baños o regaderas que las personas } \\
\text { viviendo con el VIH utilizan }\end{array}$ \\
\hline El VIH se trasmite por la picadura de un insecto \\
\hline El VIH se trasmite sólo durante la fase de ventana o VIH positivos \\
\hline $\begin{array}{l}\text { El VIH se trasmite al compartir cucharas, tenedores, platos, tazas o vasos con una persona } \\
\text { infectada }\end{array}$ \\
\hline $\begin{array}{l}\text { El VIH se trasmite al utilizar el mismo cepillo de dientes y rasuradora que ha utilizado } \\
\text { una persona infectada }\end{array}$ \\
\hline El preservativo o condón es un método eficaz para evitar la transmisión del VIH/SIDA \\
\hline $\begin{array}{l}\text { La fidelidad y la abstinencia son dos comportamientos eficaces para evitar la transmisión } \\
\text { del VIH/SIDA }\end{array}$ \\
\hline $\begin{array}{l}\text { Las poblaciones móviles, mantienen conductas de riesgo que los pueden conllevar a } \\
\text { contraer el VIH/SIDA }\end{array}$ \\
\hline $\begin{array}{l}\text { El VIH/SIDA es una infección exclusiva de los homosexuales, trabajadoras del sexo y } \\
\text { hemofílicos }\end{array}$ \\
\hline Las ITS, no están relacionadas con el VIH/SIDA \\
\hline ing
\end{tabular}


Gráfico 1. Pre y postest / conocimientos de conceptos básicos en personal de salud

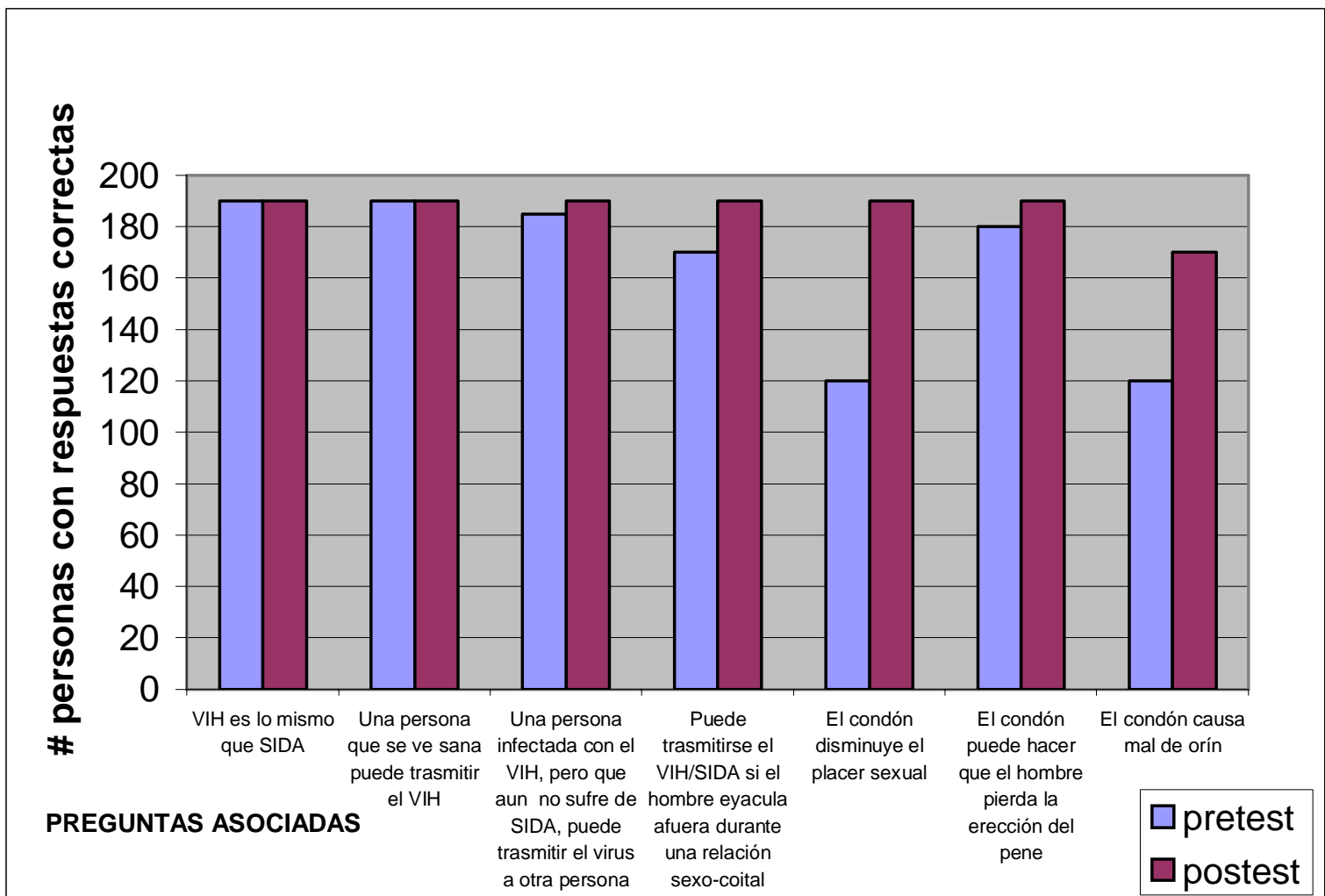

Fuente: Informe Final de Intervención en Poblaciones Móviles, El Salvador Junio 2006 


\section{Grafico 2. Pre y post/ conocimientos básicos en "el resto"}

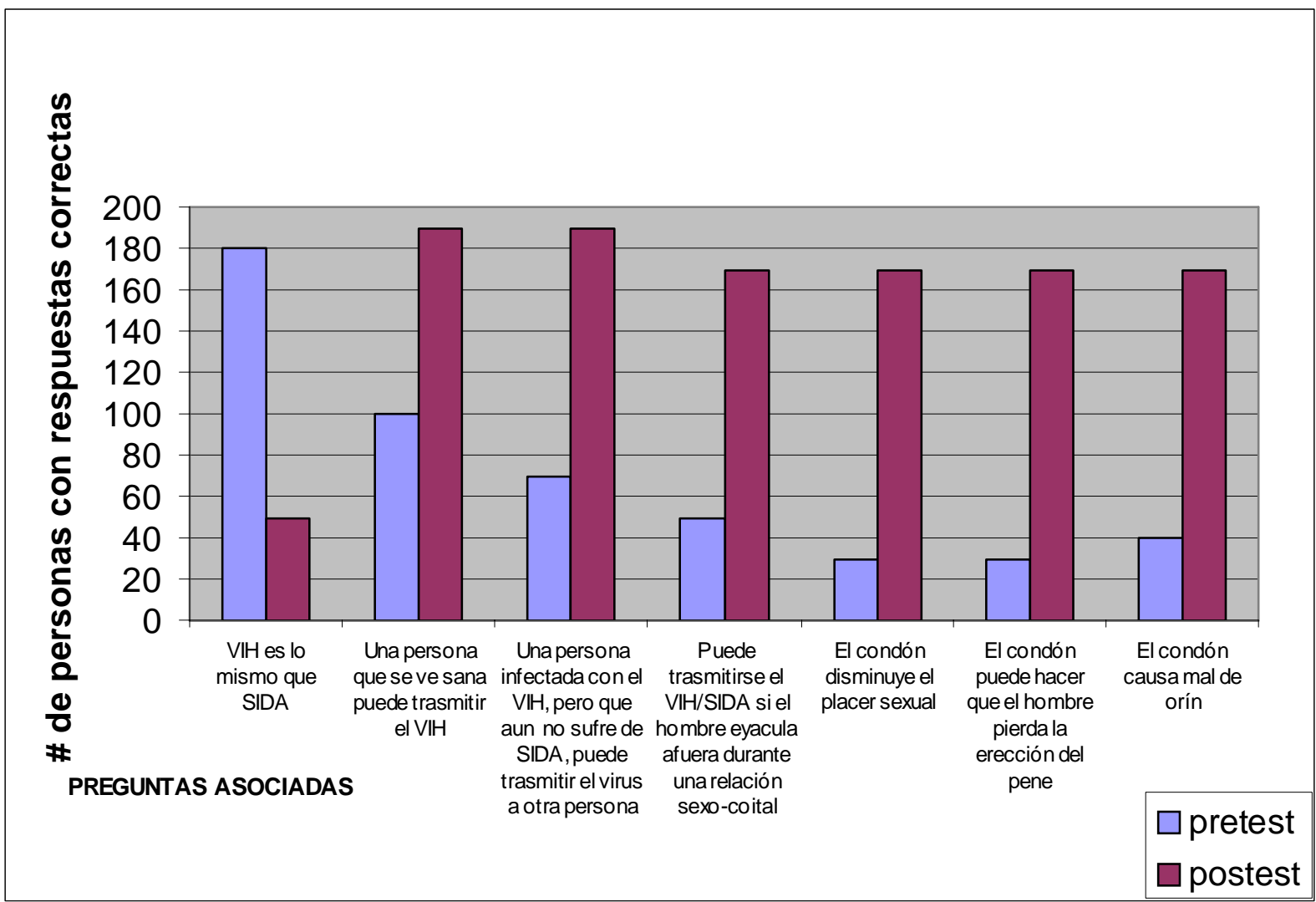

Fuente: Informe Final de Intervención en Poblaciones Móviles, El Salvador Junio 2006 


\section{Grafico 3. Estigma y discriminación en personal de salud}

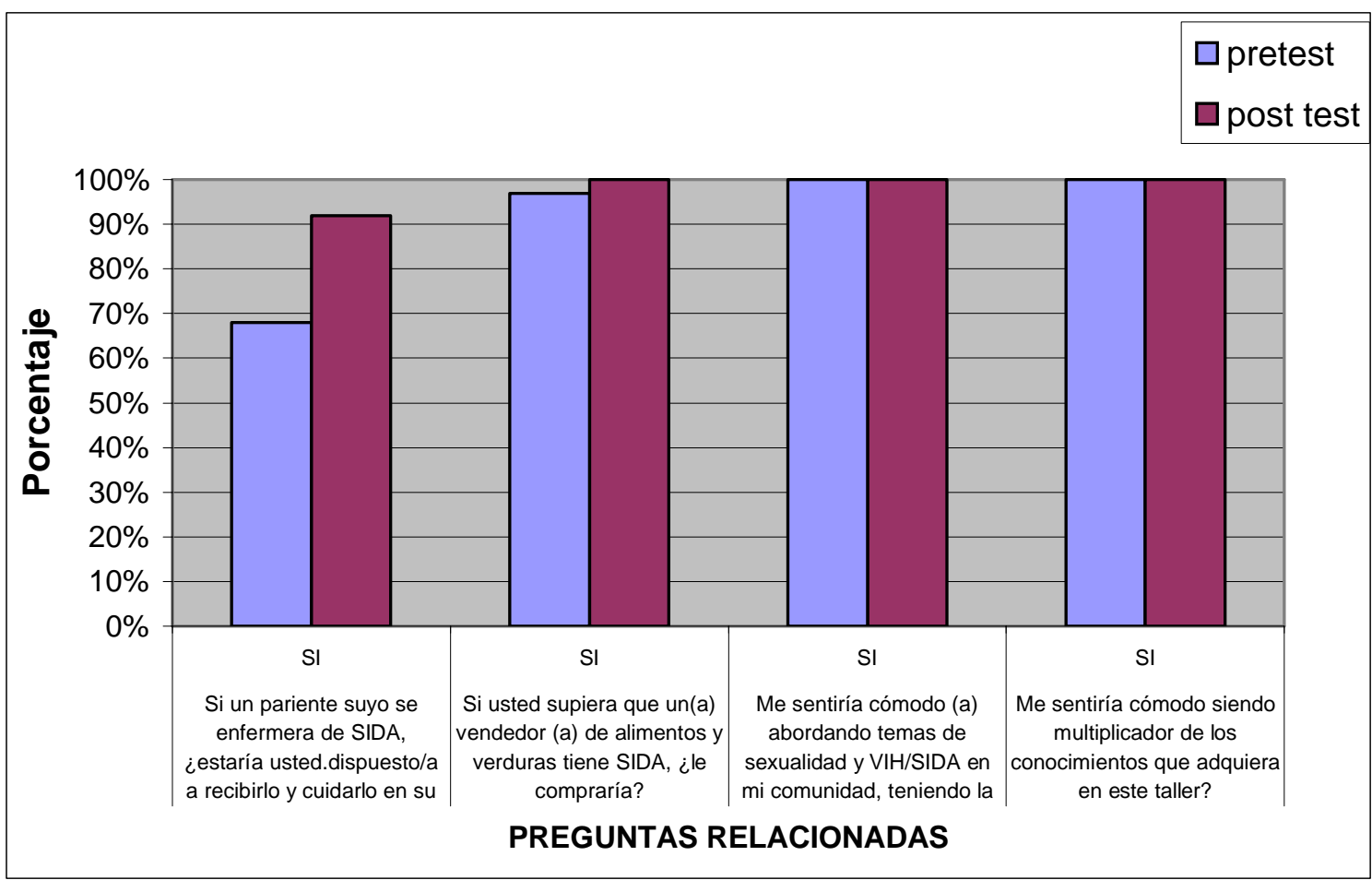

Fuente: Informe Final de Intervención en Poblaciones Móviles, El Salvador Junio 2006

\section{Grafico 4. Estigma y discriminación "el resto"}

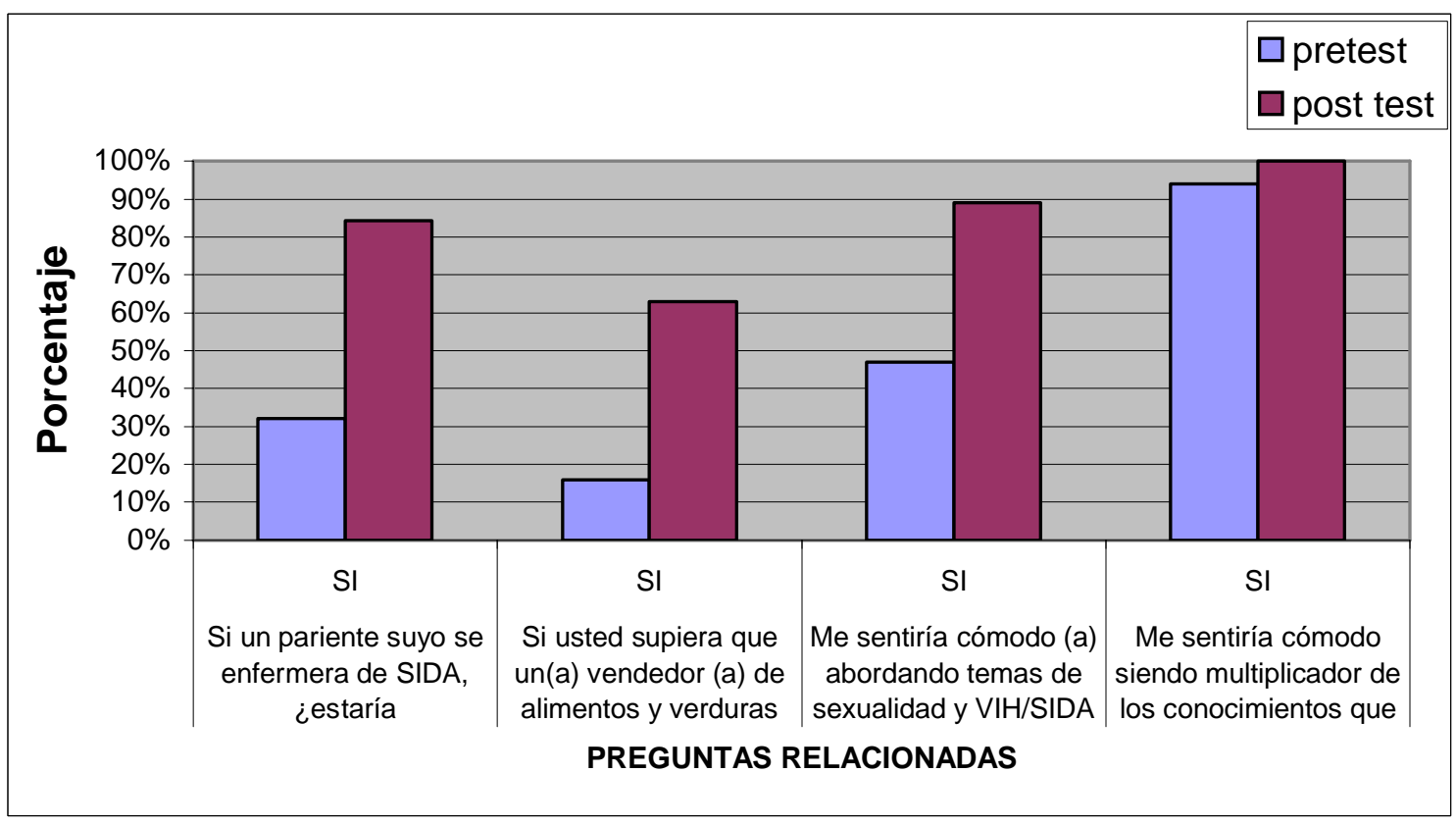

Fuente: Informe Final de Intervención en Poblaciones Móviles, El Salvador Junio 2006 


\section{Grafico 5. Pretest/ mitos y tabues sobre el vih-sida "el resto"}

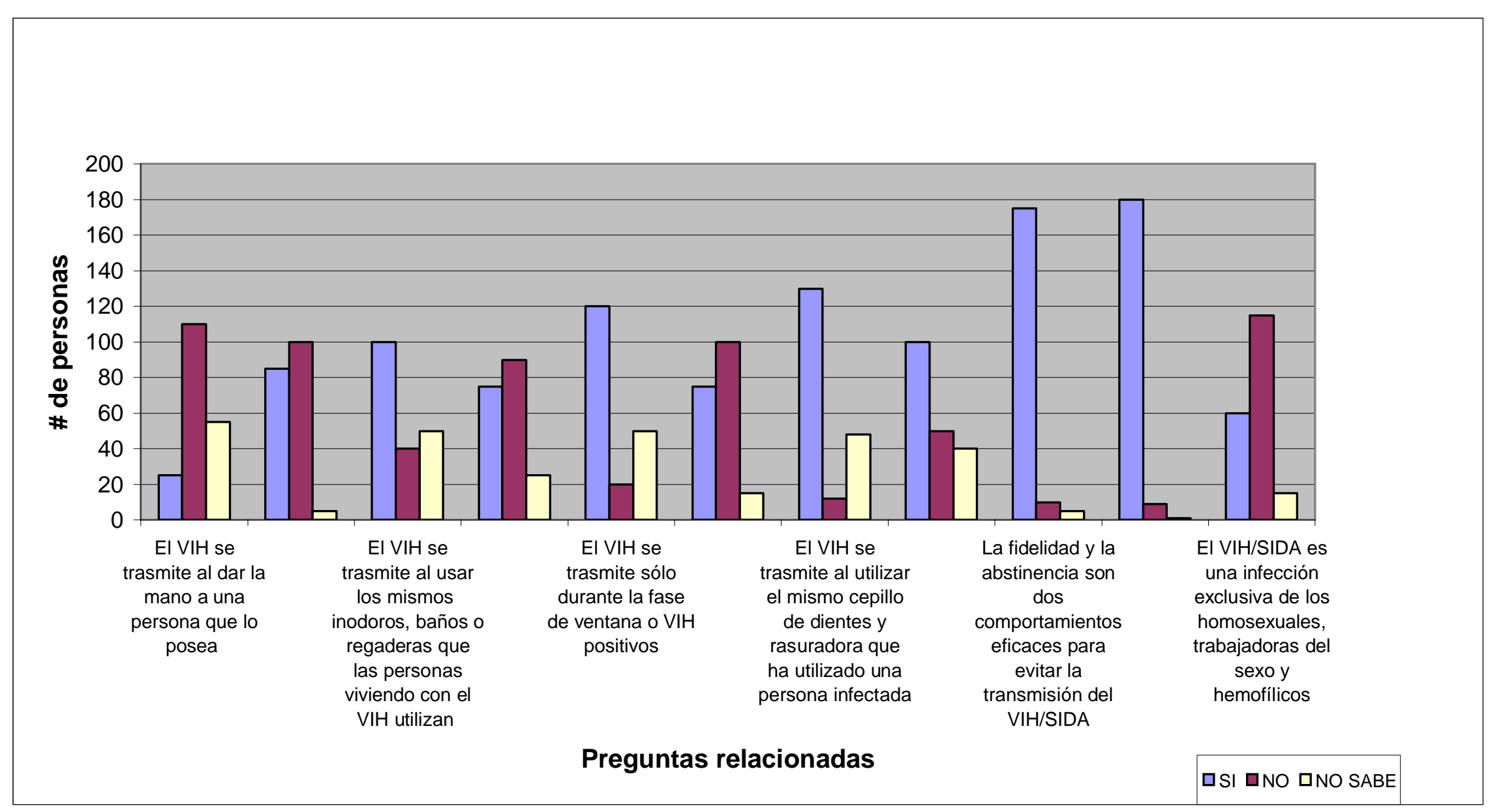

Fuente: Informe Final de Intervención en Poblaciones Móviles, El Salvador Junio 2006 


\section{Grafico 6. Postest mitos y tabues sobre vih-sida "el resto"}

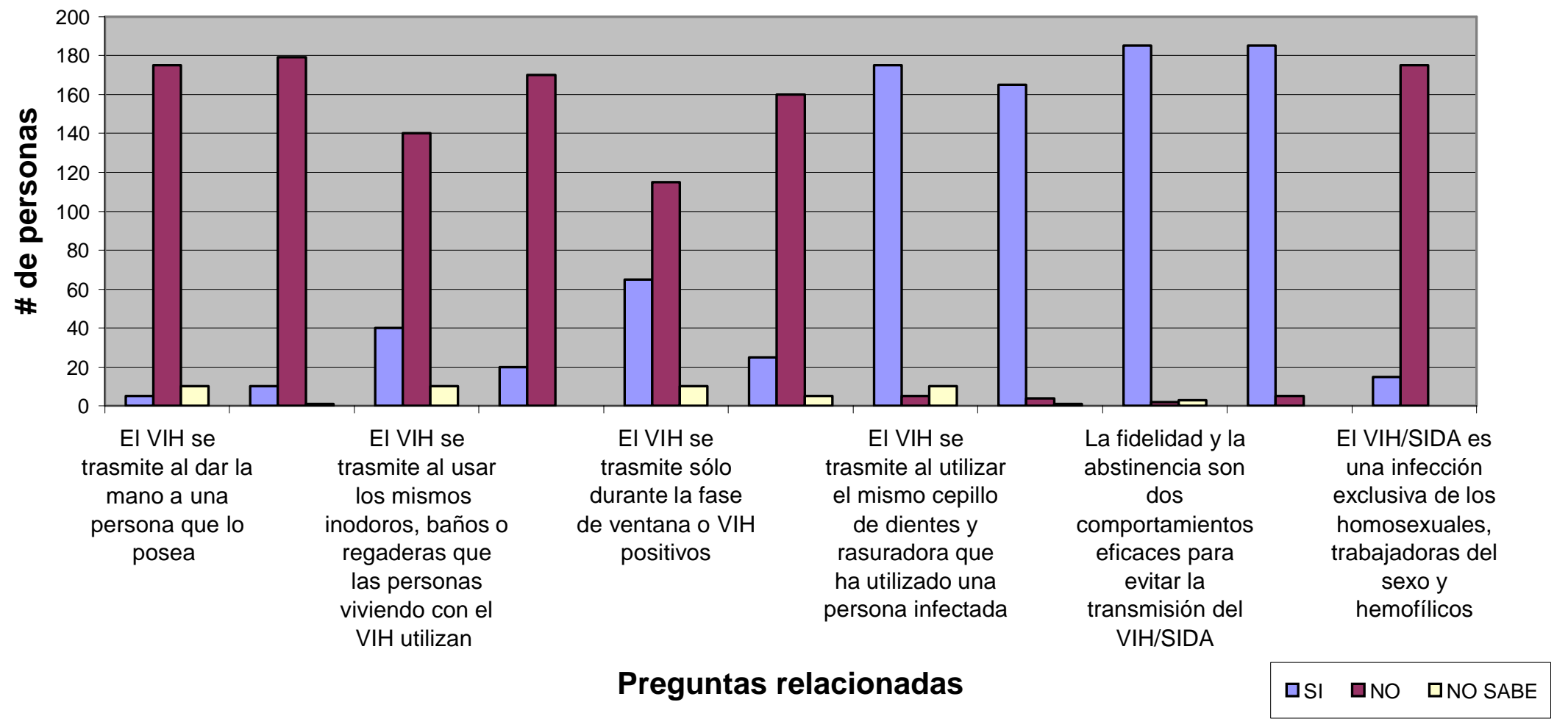

Fuente: Informe Final de Intervención en Poblaciones Móviles, El Salvador Junio 2006 